\title{
BLADDER CANCER
}

\section{Sarcopenia predicts cystectomy complications in women}

Preoperative sarcopenia in women can predict risk of complications following cystectomy, according to new research from the University of North Carolina at Chapel Hill, USA. "This is the first study, to our knowledge, to describe sarcopenia as a risk factor in the cystectomy population," Angela Smith, corresponding author of the study, told Nature Reviews Urology.

Sarcopenia is broadly defined as the degenerative loss of skeletal mass in association with ageing, and is commonly considered to be a component of frailty assessment. Given that cystectomy is largely performed in an elderly population, researchers wanted to understand the relationship between sarcopenia and postoperative morbidity in patients undergoing this complicated surgery.

Consistent with previous studies of patients undergoing other types of surgery, Smith and her colleagues evaluated sarcopenia by measuring the crosssectional area of the left and right psoas muscles on CT using 3D image analysis software. They adjusted the muscle areas according to patient height to generate a 'sarcopenia score' for each patient.

Researchers examined their institutional database to identify 200 patients who had undergone radical cystectomy, who received abdominal CT within 1 month of surgery and for whom data was available on postoperative complications. Mean sarcopenia scores were $712 \mathrm{~cm}^{2} / \mathrm{m}^{2}$ and $571 \mathrm{~cm}^{2} / \mathrm{m}^{2}$ for men and women, respectively.

Overall, sarcopenia scores were not associated with the presence of major complications; however, when the investigators assessed men and women separately they found a striking association for women only. Major complications were reported in $43 \%$ of sarcopenic women compared with 10\% of women without sarcopenia.

"If sarcopenia is an independent risk factor for morbidity in a subset of patients, clinicians can appropriately counsel these individuals and potentially

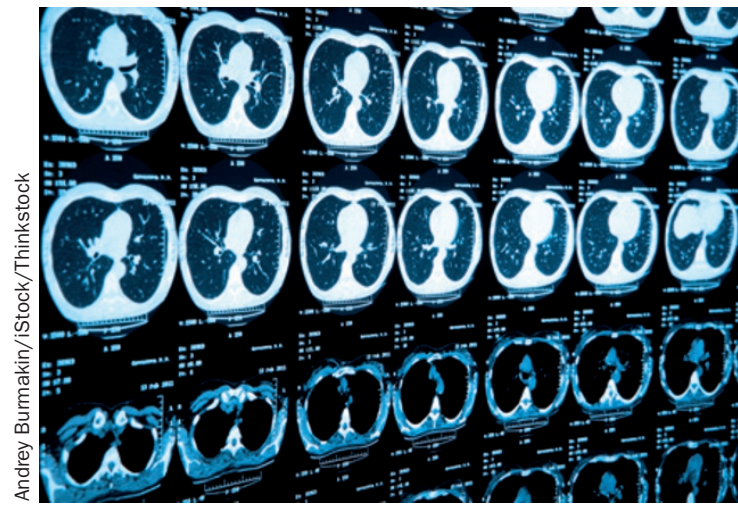

identify candidates for the less morbid trimodality approach of chemoradiation," explains Smith. "Future research will be needed to corroborate the findings of this retrospective study."

Sarah Payton

Original article Smith, A. B. et al. Sarcopenia as a predictor for complications and survival following radical cystectomy. J. Urol. doi:10.1016/j.juro.2013.12.047 